\title{
Modulatory Effects of Astragalus Polysaccharides on T-Cell Polarization in Mice with Polymicrobial Sepsis
}

\author{
Yu-Chen Hou, ${ }^{1,2}$ Jin-Ming Wu, ${ }^{1}$ Ming-Yang Wang, ${ }^{1}$ Ming-Hsun Wu, \\ Kuen-Yuan Chen, ${ }^{1}$ Sung-Ling Yeh, ${ }^{2}$ and Ming-Tsan Lin $^{1,3}$ \\ ${ }^{1}$ Department of Surgery, National Taiwan University Hospital, Taipei 100, Taiwan \\ ${ }^{2}$ School of Nutrition and Health Sciences, Taipei Medical University, Taipei 110, Taiwan \\ ${ }^{3}$ Department of Medical Education and Bioethics, College of Medicine, National Taiwan University, Taipei 100, Taiwan \\ Correspondence should be addressed to Ming-Tsan Lin; linmt@ntu.edu.tw
}

Received 13 July 2015; Revised 16 October 2015; Accepted 2 November 2015

Academic Editor: Simi Ali

Copyright (C) 2015 Yu-Chen Hou et al. This is an open access article distributed under the Creative Commons Attribution License, which permits unrestricted use, distribution, and reproduction in any medium, provided the original work is properly cited.

\begin{abstract}
Background. This study evaluated the impact of different doses of Astragalus polysaccharides (APS) on the functional status and phenotype of $\mathrm{T}$ cells during polymicrobial sepsis. Methods. On day 1 after cecal ligation and puncture, mice were treated with either saline, 100 (A100), 200 (A200), or $400 \mathrm{mg}$ APS/kg body weight (BW) (A400) by an intraperitoneal injection daily for 4 days. All mice were sacrificed 5 days after the operation. Results. APS treatment reversed the sepsis-induced decrement in the T helper (Th) cell population, and the percentage of activated Th cells also increased in the spleen and Peyer's patches. APS administration downregulated the percentages of circulating Th2 cells and regulatory T cells (Treg), and the percentage of Th17 cells in blood was upregulated in the A400 group. Weight loss and kidney injury were attenuated in the A100 and A200 groups but not in the A400 group at the end of the study. Conclusions. Treatments with 100 and $200 \mathrm{mg}$ APS $/ \mathrm{kg}$ BW reduced Treg populations and elicited a more-balanced $\mathrm{Th} 1 / \mathrm{Th} 2$ response that consequently attenuated immunosuppression in polymicrobial sepsis. High-dose APS administration led to excessive responses of Th17 cells which may have adverse effects in sepsis-induced organ injury.
\end{abstract}

\section{Introduction}

Sepsis is a characteristic set of systemic inflammatory responses to bacterial infection. Despite effective treatments with antibiotics and fluid resuscitation, morbidity and mortality from sepsis still remain high in intensive care units [1]. Sepsis activates both pro- and anti-inflammatory immune responses and causes disturbance of the immune system, characterized by a net response of initial hyperinflammation which then enters a persistent immunosuppressive phase [2]. Organ dysfunction caused by the overwhelming inflammation is the most lethal complication of sepsis [3]. Sepsisinduced immunosuppression results in failure to control primary and secondary hospital-acquired infections [4]. Balancing pro- and anti-inflammatory responses has therefore become a potential therapeutic approach for sepsis [2].

Sepsis causes a marked apoptosis-induced depletion of lymphocytes, leading to immunosuppression $[5,6]$. The prolonged duration of sepsis enhances the development of T-cell exhaustion which is correlated with nosocomial infections and mortality in septic patients [7]. $\mathrm{CD} 4^{+} \mathrm{T}$ cells, including $\mathrm{T}$ helper (Th) cells and regulatory $\mathrm{T}$ cells (Treg), play important roles in immune homeostasis during sepsis [8]. Th cells have been characterized into Th1, Th2, and Thl7 cell subsets according to the types of cytokines excreted after stimulation. Th1 and Th17 cells protect against pathogen infections by, respectively, promoting the killing ability of macrophages and neutrophils. Th 2 cells are considered to be a less protective subset during sepsis due to their enhancement of humoral immunity and inhibition of classical inflammation. Treg are implicated in immunosuppressive properties of $\mathrm{T}$ cells and innate immune cells [9]. An increased percentage of circulating Treg were found in septic patients [10], and excessive Treg contribute to lymphocyte anergy in sepsis [11].

The dried root of Astragalus membranaceus is thought to tone the vital energy [12], and it has been used as a health-promoting herb for centuries in Asia. Modern 
research revealed that the active constituents of Astragalus include polysaccharides, saponins, flavonoids, amino acids, and trace elements [13]. Astragalus polysaccharide (APS), the major component obtained from water extraction, was demonstrated to be the pharmacological component that acts as an immunopotentiator $[14,15]$ and showed suppressive effects on Treg in burned mice with bacterial infections [16]. Also, APS was found to promote a shifting of splenic CD $4^{+}$ $\mathrm{T}$ cells from a Th2 to a Th1 cytokine-producing profile in an in vitro study [17]. However, the modulatory effects of APS on T-cell polarization in polymicrobial sepsis remain unclear. Therefore, we investigated the functional status and phenotype of $\mathrm{T}$ cells from the circulation and lymphoid organs to evaluate the impacts of different doses of APS administered to control immune homeostasis during sepsis.

\section{Materials and Methods}

2.1. Animals. $\mathrm{C} 57 \mathrm{Bl} / 6 \mathrm{~J}$ male mice at 6 8-week-old and weighing 19 21 $\mathrm{g}$ at the beginning of the experiment were used in this study. Mice were purchased from the National Laboratory Animal Center (Taipei, Taiwan) and were housed in a conventional animal facility. All mice were given free access to water and laboratory chow throughout the study. This study was carried out in Taipei Medical University. Animal care and experimental procedures were reviewed and approved by the Institutional Animal Care and Use Committee of Taipei Medical University (approval number LAC-101-0284). All animal experiments were carried out according to the approved protocols. Humane endpoints were considered in this experiment. Mice would be euthanized when showing signs associated with a moribund state, including unconsciousness with no response to external stimuli, intractable seizures, labored breathing, cyanosis, inability to ambulate, and inability to eat or drink.

2.2. Experimental Design. After 1 week of acclimation, mice were randomly assigned to receive either a sham operation $(n=10)$ or cecal ligation and puncture (CLP) $(n=44)$. The CLP surgery was used to induce polymicrobial sepsis. A combination of ketamine $(80 \mathrm{mg} / \mathrm{kg})$ and xylazine $(10 \mathrm{mg} / \mathrm{kg})$ via intraperitoneal (i.p.) injection was used as the anesthetic and analgesic agents. Briefly, under anesthesia, the cecum was exposed, and cecal ligation was performed at approximately $50 \%$ of the length of the cecum with 3-0 silk. The distal cecum was then punctured twice with a 22 -gauge needle, and a small amount of feces was squeezed out through the perforations. After replacing the cecum back into the abdominal cavity, the musculature and skin were, respectively, closed using 3-0 silk sutures. Sham-operated mice were handled in the same manner except that no ligation or puncture of the cecum was performed. All mice were treated with the antibiotic Ertapenem (INVANZ, Merck, Whitehouse Station, NJ, USA) at a dose of $75 \mathrm{mg} / \mathrm{kg}$ body weight (BW) via i.p. injection. Antibiotic treatments were begun $6 \mathrm{~h}$ after surgery, and an injection was given every $24 \mathrm{~h}$ until day 2 after surgery.

On day 1 after surgery, CLP-operated mice were randomly divided into 4 groups ( $n=11$ in each group) as follows: sepsis with normal saline treatment (saline group),

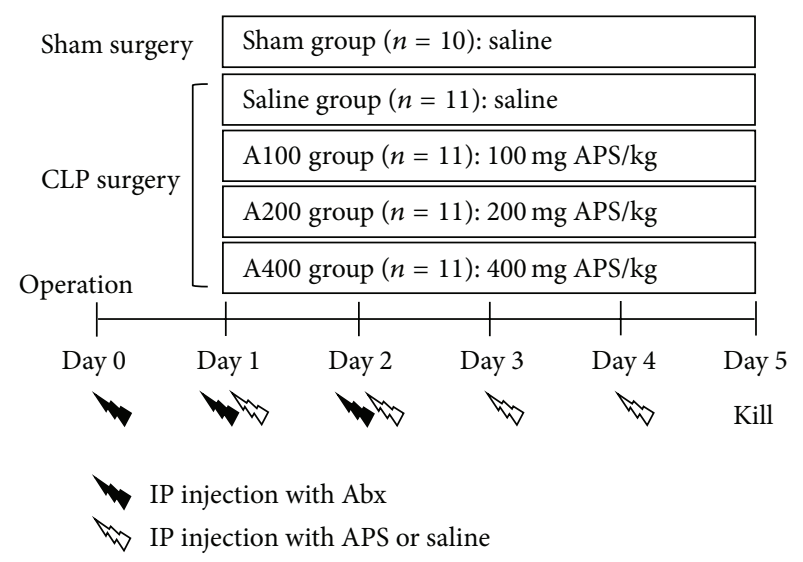

FIgURE 1: Schematic diagram of the experimental procedure. Abx: antibiotics. APS: Astragalus polysaccharides.

sepsis with $100 \mathrm{mg}$ APS $/ \mathrm{kg}$ BW treatment (A100 group), sepsis with $200 \mathrm{mg} \mathrm{APS} / \mathrm{kg} \mathrm{BW}$ treatment (A200 group), and sepsis with $400 \mathrm{mg}$ APS/kg BW treatment (A400 group). Septic mice received an i.p. injection of APS reconstituted with normal saline daily for 4 days, while mice in the sham and saline groups were given an equivalent volume of normal saline. A flow diagram of the experimental procedures is shown in Figure 1. The APS used in this study were a sterile injectable extract of Astragalus membranaceus (PhytoHealth, Taipei, Taiwan). The APS consisted of $\alpha-1,4(1,6)$ glucan, arabinose-galactose polysaccharide, rhamnose-galacturonic acid polysaccharide, and arabinosegalactose protein polysaccharide with molecular weights of 20,000 60,000 [17].

BWs and survival rates were recorded daily during the experimental period. On day 5 after the sham or CLP surgery, all mice were killed under anesthesia by cardiac puncture, and fresh blood was collected in heparinized tubes. Peyer's patches (PPs) and the spleen from each animal were removed, and the spleen was cut in half. One-half of a spleen was saved for a flow cytometric analysis, and the other half was stored at $-80^{\circ} \mathrm{C}$ for further measurements. To obtain intestinal lavage fluid (ILF), $5 \mathrm{~cm}$ of the small bowel proximal to the ileocecal valve, corresponding to the ileum, was excised and cut open longitudinally. The specimen was immersed in $1 \mathrm{~mL}$ of ice-cold phosphate-buffered saline with a protease inhibitor cocktail (Sigma, St. Louis, MO, USA) for $10 \mathrm{~min}$. The immersion fluids with luminal contents were collected and centrifuged for $10 \mathrm{~min}$ at $300 \times \mathrm{g}$. Supernatants were transferred to microfuge tubes and stored at $-80^{\circ} \mathrm{C}$ for further analysis.

2.3. Lymphocyte Characterization. Blood, spleens, and PPs were used to analyze lymphocyte populations by flow cytometry. Cell suspensions from PPs and spleens were obtained by passing the tissues through a nylon cell strainer with a $40 \mu \mathrm{m}$ pore size (BD Biosciences, San Jose, CA, USA) in RPMI1640 medium (Biological Industries, Kibbutz Beit Haemek, Israel). After lysing erythrocytes and centrifugation at $300 \times \mathrm{g}$ for $10 \mathrm{~min}$, pelleted cells were suspended in staining buffer. 
Whole-blood samples and cell suspensions from spleens and PPs were split into $100 \mu \mathrm{L}$ aliquots and incubated with PerCP-conjugated anti-CD45 (Clone 30-F11, Biolegend, San Diego, CA, USA), APC-conjugated anti-CD3ع (Clone 1452C11, eBioscience, San Diego, CA, USA), PE-conjugated antiCD19 (Clone 6D5, Biolegend), FITC-conjugated anti-CD4 (Clone GK1.5, eBioscience), and Pacific blue-conjugated antiCD8 (Clone 53-6.7, Biolegend) antibodies (Abs). Stained cells were analyzed with a FACS Canto II flow cytometer (BD Biosciences). CD45-positive cells were gated and considered to be leukocytes. Lymphocyte populations were determined as the percentages of $\mathrm{T}$ cells $\left(\mathrm{CD} 3 \varepsilon^{+} \mathrm{CD} 19^{-}\right)$and $\mathrm{B}$ cells $\left(\mathrm{CD} 3 \varepsilon^{-} \mathrm{CD} 19^{+}\right)$among leukocytes. Subpopulations of Th cells and cytotoxic $\mathrm{T}$ cells are presented as the percentage of $\mathrm{CD}^{+}{ }^{+} \mathrm{CD} 8^{-}$and $\mathrm{CD} 4^{-} \mathrm{CD} 8^{+}$cells among $\mathrm{CD} 3 \varepsilon$-expressing cells.

2.4. Activation of $T$ Lymphocytes. Expression of the activation marker, CD69, on T lymphocytes was measured by flow cytometry. Aliquots at $100 \mu \mathrm{L}$ of cell suspensions from spleens and PPs were incubated with PerCP-conjugated antiCD45, APC-conjugated anti-CD3e, FITC-conjugated antiCD4, Pacific blue-conjugated anti-CD8, and PE-conjugated anti-CD69 (Biolegend) Abs. Stained cells were run through the flow cytometer. CD45-positive leukocytes were gated, and T-cell subsets were determined as described above. Activated T lymphocytes were evaluated by the expression of CD69 of T-cell subpopulations.

2.5. Subsets of CD4-Positive T Cells in Blood. To determine the phenotypes of Th cells, $100 \mu \mathrm{L}$ aliquots of blood were incubated with a Pacific blue-conjugated anti-CD4 (BD Biosciences) Ab, fixed, and then permeated for intracellular cytokine staining. The following Abs were used for intracellular cytokine staining: Alexa Fluor 488-conjugated anti-interleukin- (IL-) 4 (Biolegend), APC-conjugated antiinterferon- (IFN-) $\gamma$ (BD Biosciences), and PE-conjugated anti-IL-17A (Biolegend) Abs. To analyze Treg, $100 \mu \mathrm{L}$ aliquots of blood were incubated with Pacific blue-conjugated antiCD4 and APC-conjugated anti-CD25 (eBioscience) Abs. After incubation for $30 \mathrm{~min}$, leukocytes were fixed and permeated with Foxp3 staining buffer (eBioscience). PEconjugated anti-Foxp3 (Biolegend) Abs were then added for the intracellular staining of Foxp3.

After staining, red blood cells were lysed, and leukocytes were analyzed by flow cytometry. CD4-positive lymphocytes were gated on the basis of low forward and side scatter. Phenotypes of Th cells are presented as percentages of Thassociated cytokine-expressing cells in CD4-positive lymphocytes. Treg populations are presented as a percentage of CD25/Foxp3 double-positive cells in gated CD4-expressing lymphocytes.

2.6. Real-Time Quantitative Polymerase Chain Reaction ( $q P C R)$. RNA extraction from spleens was prepared with the TRIzol reagent (Invitrogen, Carlsbad, CA, USA), and complementary (c)DNA was synthesized from $1 \mu \mathrm{g}$ of total RNA using a cDNA synthesis kit (Fermentas, Glen Burnie, $\mathrm{MD}$, USA) according to the manufacturers' instructions.
Expressions of cytokine genes in spleens were analyzed using the ABI 7300 qPCR System (Applied Biosystems, Foster City, CA, USA). Amplification was performed in a total volume of $25 \mu \mathrm{L}$ containing $5 \mu \mathrm{L}$ of $1 / 100$ diluted cDNA, $100 \mathrm{nM}$ of each primer, and $1 \mathrm{X}$ SYBR green master mix reagent (Applied Biosystems) using the thermocycling protocol recommended by the PCR system. Primer sequences were as follows: mouse IFN- $\gamma\left(5^{\prime}\right.$-ATGAACGCTACACACTGCATC$3^{\prime}$ and $5^{\prime}$-CCATCCTTTTGCCAGTTCCTC- $\left.3^{\prime}\right)$, IL-4 (5' ACAGGAGAAGGGACGCCAT- $3^{\prime}$ and $5^{\prime}$-GAAGCCCTACAGACGAGCTCA-3 $\left.{ }^{\prime}\right)$, IL-17A (5'-AGCAAGAGATCCTGGTCCTGAA- $3^{\prime}$ and $5^{\prime}$-CATCTTCTCGACCCTGAAAGTGA-3 $\left.{ }^{\prime}\right)$, Foxp3 (5'-AGCGAGTGTCCCTGCTCTCCC$3^{\prime}$ and $5^{\prime}$-CTTCTGTCTGGAGTGGCTGGGTGT-3'), IL-6 ( $5^{\prime}$-GGGACTGATGCTGGTGACAA- $3^{\prime}$ and $5^{\prime}$-ACAGGTCTGTTGGGAGTGGT-3'), IL-2 (5'-AACCTGAAACTCCCCAGGAT- $3^{\prime}$ and $5^{\prime}$-TCATCGAATTGGCACTCAAA$\left.3^{\prime}\right)$, transforming growth factor (TGF)- $\beta$ ( $5^{\prime}$-GCCCTGGATACCAACTATTGCTT- $3^{\prime}$ and $5^{\prime}$-AGTTGGCATGGTAGCCCTTG- $\left.3^{\prime}\right)$, and $\beta$-actin $\left(5^{\prime}\right.$-ACCCACACTGTGCCCATCTAC- $3^{\prime}$ and $5^{\prime}$-TCGGTGAGGATCTTCATGAGGTA$\left.3^{\prime}\right)$. All samples were analyzed in triplicate, and multiples of change in messenger (m)RNA were calculated by the equation $2^{-\Delta \Delta \mathrm{Ct}}$ ( $\Delta \mathrm{Ct}$ indicates the difference in threshold cycles between the test gene and $\beta$-actin, and $\Delta \Delta \mathrm{Ct}$ indicates the difference in $\Delta \mathrm{Ct}$ between the CLP and sham groups).

2.7. Immunoglobulin A (IgA) Quantification in ILF. Luminal IgA was determined by an enzyme-linked immunosorbent assay kit with the corresponding capture antibody for IgA (ICL, Portland, OR, USA). Procedures followed the manufacturer's instructions. The amount of IgA was determined by detecting the antibody conjugated to horseradish peroxidase.

2.8. Histopathology. The kidneys were harvested, fixed with $4 \%$ paraformaldehyde, and embedded in paraffin. Specimens were sliced into $5 \mu \mathrm{m}$-thick sections and stained with hematoxylin-eosin (H\&E) for the histopathological analysis. Digital images at 200x magnification per section were captured with a Zeiss Axiophot light microscope (Carl Zeiss MicroImaging LLC, Thornwood, NY, USA) and a Nikon D1X digital camera (Tokyo, Japan). Five fields per section were examined to determine the morphological changes and lesions. The degree of kidney injury was measured by a semiquantitative scoring system according to Kuruş et al. [18]. The total histological score ranged from 0 to 8 , which represented the summed scores of interstitial fibrosis and tubular injury in the renal cortex (Table 1).

2.9. Statistical Analysis. Data are presented as the mean \pm standard error of the mean (SEM). Statistical analyses were performed using GraphPad Prism 5 software (GraphPad Software, San Diego, CA, USA). Survival rates were evaluated by the log-rank test. Differences among groups were analyzed by a one-way analysis of variance (ANOVA) with Tukey's test. A $p$ value of $<0.05$ was considered statistically significant. 
TABLE 1: The histological scoring system of kidney injury.

\begin{tabular}{|c|c|c|}
\hline Feature & Score & Description \\
\hline \multirow{4}{*}{ Interstitial fibrosis } & 0 & Normal interstitium and tubules \\
\hline & 1 & Mild interstitial thickening between the tubules \\
\hline & 2 & Moderate interstitial thickening between the tubules \\
\hline & 3 & Severe interstitial thickening between the tubules \\
\hline \multirow{6}{*}{ Tubular injury* $^{*}$} & 0 & No tubular injury \\
\hline & 1 & $<10 \%$ of tubules injured \\
\hline & 2 & $10 \% \sim 25 \%$ of tubules injured \\
\hline & 3 & $26 \% \sim 50 \%$ of tubules injured \\
\hline & 4 & $51 \% \sim 75 \%$ of tubules injured \\
\hline & 5 & $>75 \%$ of tubules injured \\
\hline
\end{tabular}

* Tubular injury was defined as tubular dilation, tubular atrophy, vacuolization, sloughing of tubular epithelial cells, or thickening of the tubular basement membrane.

TABLE 2: Lymphocyte populations (\%).

\begin{tabular}{|c|c|c|c|c|c|}
\hline & Sham & Saline & A100 & A200 & A400 \\
\hline \multicolumn{6}{|l|}{ Blood } \\
\hline $\mathrm{T}$ cells & $17.4 \pm 0.8$ & $13.1 \pm 1.3^{*}$ & $12.9 \pm 0.8^{*}$ & $13.0 \pm 1.0^{*}$ & $11.7 \pm 0.8^{*}$ \\
\hline Th cells & $58.4 \pm 1.0$ & $51.4 \pm 1.6^{*}$ & $51.8 \pm 1.5^{*}$ & $53.4 \pm 1.4$ & $53.7 \pm 1.4$ \\
\hline Tc cells & $32.9 \pm 2.8$ & $33.7 \pm 3.3$ & $36.0 \pm 3.7$ & $36.8 \pm 2.8$ & $35.1 \pm 3.1$ \\
\hline B cells & $49.8 \pm 1.6$ & $31.1 \pm 1.4^{*}$ & $30.2 \pm 2.6^{*}$ & $25.6 \pm 2.7^{*}$ & $22.3 \pm 3.0^{*}$ \\
\hline \multicolumn{6}{|l|}{ Spleen } \\
\hline $\mathrm{T}$ cells & $24.2 \pm 0.6$ & $20.4 \pm 1.0^{*}$ & $19.7 \pm 0.6^{*}$ & $18.9 \pm 0.7^{*}$ & $19.6 \pm 0.7^{*}$ \\
\hline Th cells & $47.6 \pm 1.3$ & $39.9 \pm 1.2^{*}$ & $42.5 \pm 0.8^{*}$ & $43.5 \pm 1.0$ & $44.1 \pm 0.8$ \\
\hline Tc cells & $34.4 \pm 0.7$ & $36.1 \pm 0.7$ & $34.0 \pm 0.6$ & $33.3 \pm 0.3$ & $34.0 \pm 1.1$ \\
\hline B cells & $47.1 \pm 1.4$ & $48.8 \pm 1.4$ & $48.9 \pm 1.4$ & $50.5 \pm 1.4$ & $49.1 \pm 1.3$ \\
\hline \multicolumn{6}{|c|}{ Peyer's patches } \\
\hline $\mathrm{T}$ cells & $8.4 \pm 0.5$ & $11.7 \pm 1.0$ & $15.6 \pm 2.4^{*}$ & $16.2 \pm 1.8^{*}$ & $17.4 \pm 2.4^{*}$ \\
\hline Th cells & $53.8 \pm 3.7$ & $28.4 \pm 1.3^{*}$ & $27.3 \pm 3.2^{*}$ & $31.7 \pm 4.1^{*}$ & $29.1 \pm 2.5^{*}$ \\
\hline Tc cells & $16.9 \pm 2.0$ & $14.4 \pm 1.7$ & $15.0 \pm 2.8$ & $16.6 \pm 2.5$ & $15.3 \pm 2.5$ \\
\hline B cells & $77.8 \pm 1.9$ & $58.1 \pm 2.4^{*}$ & $52.7 \pm 5.6^{*}$ & $52.9 \pm 5.2^{*}$ & $53.6 \pm 6.1^{*}$ \\
\hline
\end{tabular}

CD45-positive cells were gated to determine the population of lymphocytes by flow cytometry. Populations of $\mathrm{T}$ and $\mathrm{B}$ cells were determined as the percentages of $\mathrm{CD} 3 \varepsilon^{+} \mathrm{CD} 19^{-}$and $\mathrm{CD} 3 \varepsilon^{-} \mathrm{CD} 19^{+}$cells among CD45-positive cells. Percentages of $\mathrm{T}$ helper (Th) and cytotoxic $\mathrm{T}(\mathrm{Tc})$ cells were, respectively, determined by $\mathrm{CD} 4^{+} \mathrm{CD} 8^{-}$and $\mathrm{CD} 4^{-} \mathrm{CD} 8^{+}$cells in $\mathrm{CD} 3 \varepsilon$-expressing $\mathrm{T}$ lymphocytes. Values are presented as the mean $\pm \mathrm{SEM}$. ${ }^{*}$ Significantly different from the sham group $(p<0.05)$.

A100, A200, and A400, respectively, indicate treatment with 100, 200, and $400 \mathrm{mg}$ Astragalus polysaccharides/kg of body weight.

\section{Results}

3.1. Survival and BW Loss. No mortality occurred in the sham- or APS-treated groups. In the saline group, one mouse died on day 4 after CLP surgery. The mouse did not show signs associated with a moribund state before death. However, there were no significant differences in survival rates between the sham-operated and CLP mice during the study period. The initial BW among the 5 groups did not differ. BW loss was observed in the sepsis groups compared to the sham group at the end of the study. Weight loss was attenuated in the A100 and A200 groups (Figure 2).

3.2. Lymphocyte Populations in Blood and Lymphoid Tissues Were Affected by APS Treatment. Compared to shamoperated mice, percentages of circulating $\mathrm{T}$ and $\mathrm{B}$ cells decreased in septic mice. Lower percentages of $\mathrm{T}$ cells in spleens and B cells in PPs were observed in mice subjected to CLP. Reductions of Th cell subsets in the blood, spleen, and PPs were observed in the saline group (Table 2). APS treatment had no influence on the total circulating and splenic T- and B-cell populations in septic mice. However, higher percentages of T cells were observed in PPs from mice treated with APS. Populations of Th cells in the blood and spleen were maintained by treatments with 200 and $400 \mathrm{mg}$ APS $/ \mathrm{kg}$ of BW (Table 2). Representative flow cytometry plots are shown in Supplementary Figures 1 and 2 in Supplementary Material available online at http://dx.doi.org/10.1155/2015/826319.

3.3. APS Treatment Contributes to Th Cell Activation in the Spleen and PPs. There were no significant differences in activation levels of splenic $\mathrm{T}$ cells between the sham and saline groups. The percentage of activated Th cells in PPs from septic mice was lower than that from sham-operated 
TABLE 3: T lymphocyte activation (\%).

\begin{tabular}{|c|c|c|c|c|c|}
\hline & Sham & Saline & A100 & A200 & A 400 \\
\hline \multicolumn{6}{|l|}{ Spleen } \\
\hline $\mathrm{CD} 9^{+}$Th cells & $10.4 \pm 0.6$ & $12.4 \pm 1.2$ & $14.5 \pm 0.9^{*}$ & $15.3 \pm 0.8^{*}$ & $16.6 \pm 1.0^{*}$ \\
\hline $\mathrm{CD} 9^{+} \mathrm{Tc}$ cells & $4.6 \pm 0.4$ & $7.4 \pm 1.4$ & $7.0 \pm 0.8$ & $8.1 \pm 1.5$ & $8.3 \pm 2.1$ \\
\hline \multicolumn{6}{|l|}{ Peyer's patches } \\
\hline $\mathrm{CD} 9^{+}$Th cells & $53.6 \pm 2.5$ & $38.3 \pm 3.2^{*}$ & $37.7 \pm 2.5^{*}$ & $45.6 \pm 2.3$ & $46.0 \pm 4.0$ \\
\hline $\mathrm{CD} 9^{+} \mathrm{Tc}$ cells & $42.8 \pm 6.2$ & $45.3 \pm 6.8$ & $43.3 \pm 6.3$ & $42.5 \pm 5.4$ & $46.1 \pm 5.7$ \\
\hline
\end{tabular}

$\mathrm{T}$ helper (Th) and cytotoxic $\mathrm{T}(\mathrm{Tc})$ cells were, respectively, identified by $\mathrm{CD} 4^{+} \mathrm{CD} 8^{-}$and $\mathrm{CD} 4^{-} \mathrm{CD} 8^{+}$cells among $\mathrm{CD} 3 \varepsilon$-positive cells using a flow cytometer. Activated T cells were determined by the expression of CD 69 on T-cell subsets. Values are presented as the mean \pm SEM. * Significantly different from the sham group $(p<0.05)$.

A100, A200, and A400, respectively, indicate treatment with 100, 200, and $400 \mathrm{mg}$ Astragalus polysaccharides/kg of body weight.

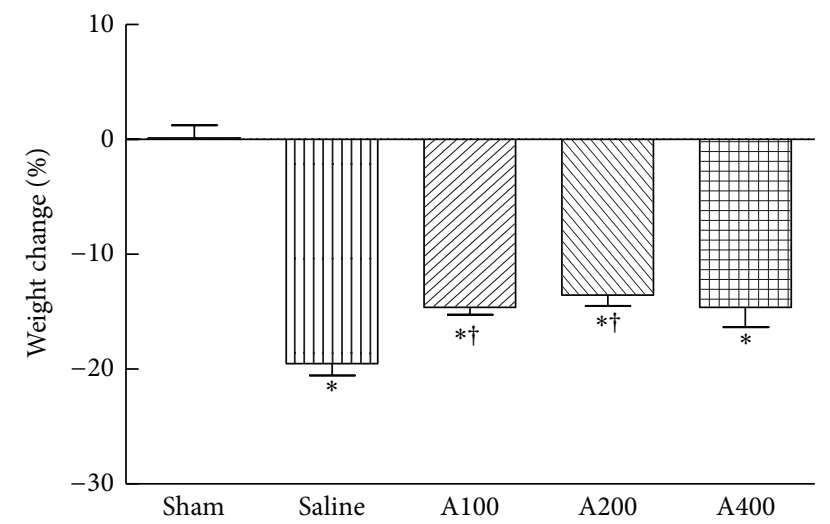

FIGURE 2: Percentage of body weight changes at the end of the study. Data are presented as the mean \pm SEM. ${ }^{*}$ Significantly different from the sham groups $(p<0.05) .{ }^{\dagger}$ Significantly different from the saline group $(p<0.05)$.

mice (Table 3). APS treatment in septic mice significantly promoted activation of splenic Th cells, and activation levels of Th cells in PPs were maintained in the A200 and A400 groups (Table 3). Representative flow cytometry plots are shown in Supplementary Figure 2.

3.4. Circulating Th2 Cells and Treg Are Suppressed by APS Treatment. There were no significant differences in percentages of IFN- $\gamma$-producing Thl cells and IL-17A-expressing Th17 cells between the sham and saline groups (Figures $3(\mathrm{a})$ and $3(\mathrm{~d})$ ). The reduction in the IFN- $\gamma /$ IL4 ratio in the saline group was accompanied by higher percentages of IL-4expressing Th2 cells (Figures 3(b) and 3(c)). The population of Treg was upregulated in the saline group compared to the sham group (Figure 3(e)). APS treatment in septic mice significantly decreased percentages of IL-4-expressing Th2 cells and the Treg population (Figures 3(b) and 3(e)). Of note, treatments with a high dose of APS elevated the IFN- $\gamma /$ IL4 ratio (Figure 3(c)) and had promotive effects on IL-17A expression by Th17 cells (Figure 3(d)). Representative flow cytometry plots are shown in Supplementary Figure 3.

3.5. Th2- and Treg-Related Genes in the Spleen Are Downregulated by APS Treatment. Compared to the sham group, mice in the saline group had lower expression levels of the IFN- $\gamma$, IL-17A, IL-6, and IL-2 genes, whereas IL-4 gene expression was upregulated. There were no significant differences in expressions of the Foxp 3 and TGF- $\beta$ genes between the sham and saline groups (Table 4). APS treatment had no effects on expressions of the IFN- $\gamma$, IL-2, and TGF- $\beta$ genes. mRNA levels of IL-4 and Foxp3 were lower in APS-treated groups. Suppression of the IL-17A and IL-6 genes in septic mice was prevented by APS administration. IL-17A and IL-6 gene expressions in the A400 group were higher than those in the sham group (Table 4 ).

3.6. APS Treatments Increase Intestinal IgA Concentrations. Compared to the sham group, luminal IgA levels gradually decreased in septic mice. Higher IgA concentrations were observed in the A200 and A400 groups than in the saline group (Figure 4).

3.7. Histopathological Aspects of the Kidneys. Morphological changes and semiquantitative scores of H\&E-stained kidney tissues are shown in Figure 5. Dilated renal tubules and swollen tubular cells were observed in septic mice. Minor kidney injuries were observed in the A100 and A200 groups (Figure 5(b)). Thickened basement membranes of renal tubules were found in mice treated with the highest dose of APS (Figure 5(a)).

\section{Discussion}

Astragalus membranaceus, with a long history of use in Chinese herbalism, has been mentioned as an immunomodulator, which was found to stimulate macrophage activity [19] and to reverse the immunosuppression seen in lung cancer patients by inhibiting Th2 polarization [20]. Among the active constituents, APS has been most widely investigated for their immunopotentiating ability, including activating mouse macrophages and stimulating the proliferation of murine splenocytes and human lymphocytes $[15,19]$. In this study, we evaluated the outcomes on day 5 after CLP in order to investigate the effects of APS on the regulatory roles of adaptive immune responses during sepsis. Our results show that APS treatments prevented the sepsis-induced decrement of the Th cell population, inhibited Th2 and Treg polarization, and potentiated Th cell activation, which was correlated with 


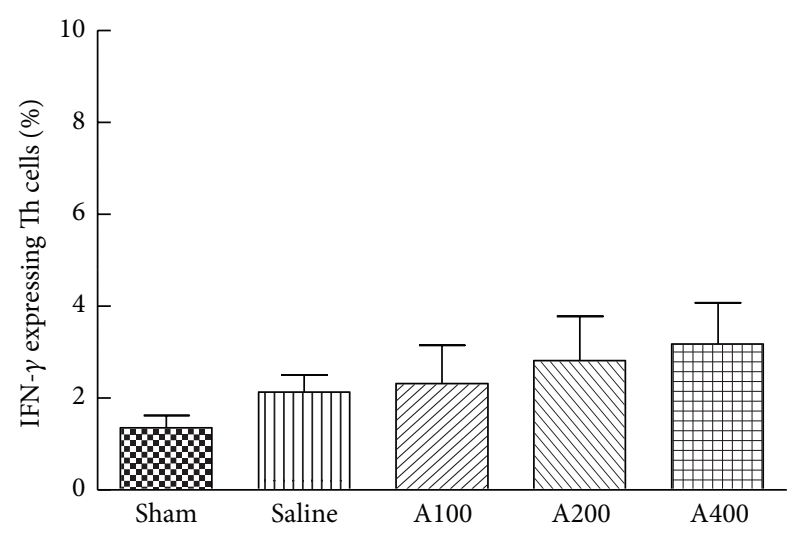

(a)

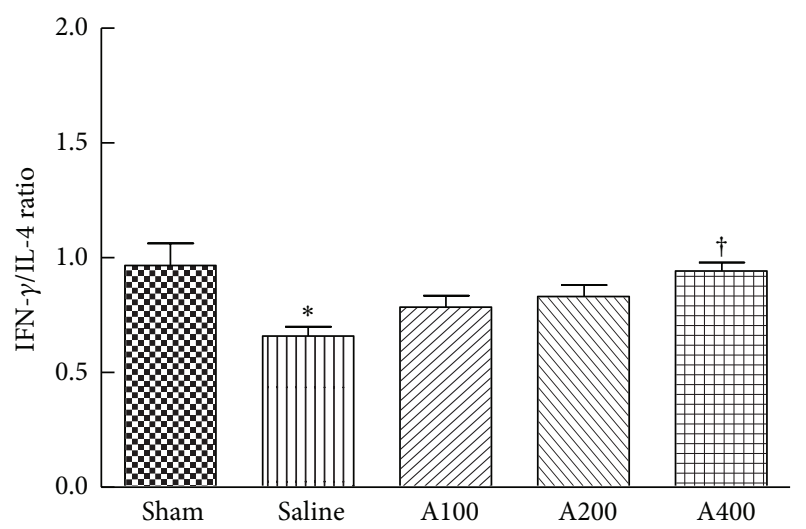

(c)

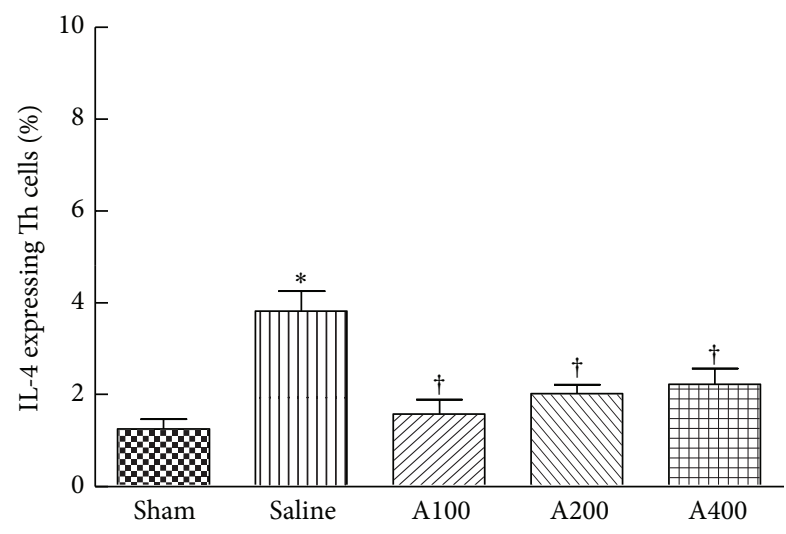

(b)

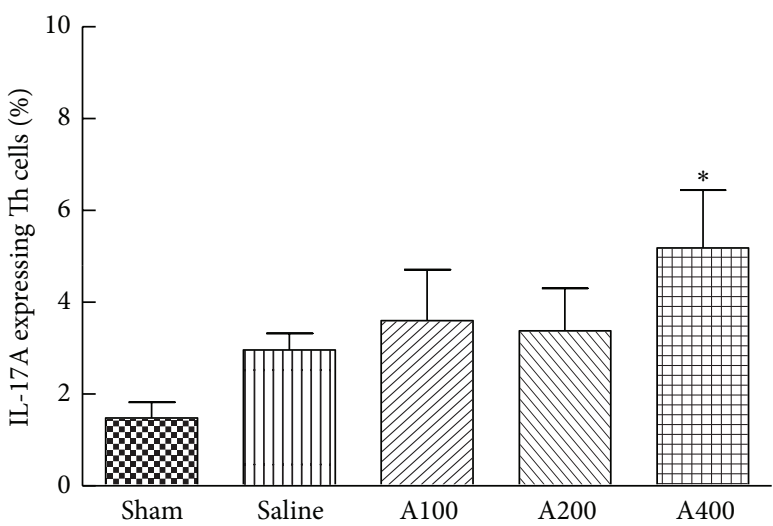

(d)

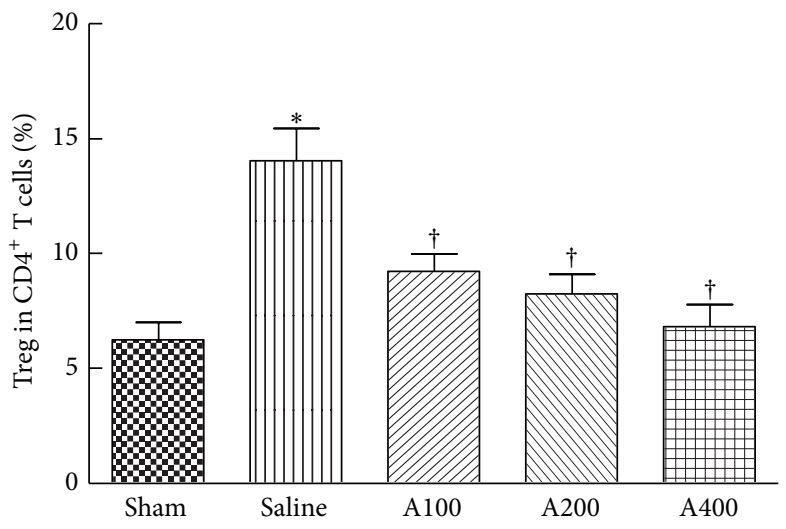

(e)

FiguRE 3: Subpopulations of CD4-positive T cells in the blood. CD4 ${ }^{+}$blood lymphocytes were gated to analyze expression levels of interferon(IFN-) $\gamma$, interleukin- (IL-) 4, and IL-17A by flow cytometry ( $\mathrm{a}, \mathrm{b}$, and $\mathrm{d}$ ). The ratio of IFN- $\gamma$ - and IL-4-expressing T helper (Th) cells was calculated (c). (e) Percentage of $\mathrm{CD}^{+}{ }^{+} \mathrm{CD} 25^{+}$Foxp $^{+}$regulatory T cells (Treg) among CD $4^{+}$lymphocytes. Data are presented as the mean \pm SEM. ${ }^{*}$ Significantly different from the sham group $(p<0.05)$. ${ }^{\dagger}$ Significantly different from the saline group $(p<0.05)$.

attenuating postseptic immunosuppression. The APS dosages used in this study were previously proven to be effective in suppressing Treg in mice under an infectious condition [16], and the low dose of APS ( $100 \mathrm{mg} / \mathrm{kg} \mathrm{BW}$ ) was equivalent to the clinical dose used to relieve cancer-related fatigue based on the body surface area normalization method [21, 22].

The CLP model is widely used in sepsis research. CLP induced lymphocyte apoptosis, which mimics human sepsis
[23]. Mortality in the CLP model is affected by the length of the cecum ligated, the needle size used for perforation, the number of punctures, and antibiotic treatments [24]. In the present study, low mortality was observed in septic mice, which was due to administration of antibiotics. According to a previous study performed in our lab, without antibiotic treatment, the mortality rate of the CLP model used in this study was approximately $45 \%$ (unpublished data). Mice were 
TABLE 4: Expression of T-cell polarization-related genes in the spleen.

\begin{tabular}{lccccc}
\hline & Sham & Saline & A100 & A200 & A400 \\
\hline & & \multicolumn{2}{c}{ Relative mRNA ratio } & & \\
IFN- $\gamma$ & $1.07 \pm 0.06$ & $0.33 \pm 0.03^{*}$ & $0.42 \pm 0.10^{*}$ & $0.46 \pm 0.03^{*}$ & $0.42 \pm 0.08^{*}$ \\
IL-4 & $1.06 \pm 0.04$ & $2.52 \pm 0.20^{*}$ & $1.55 \pm 0.20^{\dagger}$ & $1.52 \pm 0.17^{\dagger}$ & $1.13 \pm 0.26^{\dagger}$ \\
IL-17A & $1.06 \pm 0.06$ & $0.43 \pm 0.07^{*}$ & $0.71 \pm 0.07$ & $0.72 \pm 0.12$ & $0.90 \pm 0.17^{\dagger}$ \\
Foxp3 & $1.00 \pm 0.07$ & $0.78 \pm 0.07$ & $0.53 \pm 0.06^{*}$ & $0.54 \pm 0.02^{*}$ & $0.55 \pm 0.11^{*}$ \\
IL-6 & $1.00 \pm 0.02$ & $0.45 \pm 0.03^{*}$ & $0.92 \pm 0.12$ & $0.96 \pm 0.12$ & $1.33 \pm 0.23^{\dagger}$ \\
IL-2 & $1.02 \pm 0.10$ & $0.53 \pm 0.08^{*}$ & $0.57 \pm 0.10^{*}$ & $0.65 \pm 0.05^{*}$ & $0.64 \pm 0.08^{*}$ \\
TGF- $\beta$ & $1.00 \pm 0.09$ & $0.79 \pm 0.07$ & $0.92 \pm 0.09$ & $0.83 \pm 0.04$ & $0.98 \pm 0.08$ \\
\hline
\end{tabular}

The relative amount of mRNA was calculated using the comparative $\mathrm{Ct}$ method, and mRNA expression of sham-operated mice was used as a calibrator. Data are presented as the mean \pm SEM. ${ }^{*}$ Significantly different from the sham group $(p<0.05)$. ${ }^{\dagger}$ Significantly different from the saline group $(p<0.05)$. A100, A200, and A400, respectively, indicate treatment with 100, 200, and $400 \mathrm{mg}$ Astragalus polysaccharides/kg of body weight.

IFN: interferon; IL: interleukin; TGF: transforming growth factor.

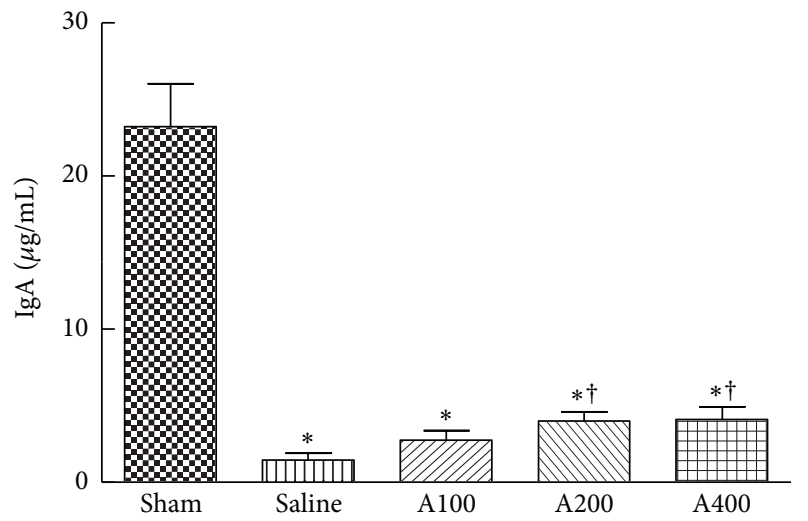

FIGURE 4: IgA concentration in intestinal lavage fluid. Data are presented as the mean \pm SEM. * Significantly different from the sham groups $(p<0.05)$. ${ }^{\dagger}$ Significantly different from the saline group $(p<0.05)$.

given antibiotics after CLP surgery to imitate a common medical treatment in septic patients, which is more clinically relevant.

Previous studies showed that sepsis results in significant decrements in T lymphocytes and CD4 cell numbers and impairment of T-cell functions [8]. Sepsis-induced apoptosis causes depletion of immune cells leading to immunosuppression [2], and apoptosis of immune cells occurs in lymphoid tissues and gut-associated lymphoid tissues [25]. In this study, we analyzed lymphocytes from the blood, spleen, and PPs, because lymphocytes from the systemic circulation and lymphoid organs are important in regulating the homeostasis of immune function during sepsis. The spleen performs the important function of removing blood-borne pathogens from the circulation. Naive $\mathrm{T}$ cells are activated by circulating antigens in the spleen and differentiate into different effector subsets. Intestinal PPs are essential for mucosal adaptive immunity. Our results indicated that sepsis caused decrements in circulating $\mathrm{T}$ and $\mathrm{B}$ lymphocytes, reductions of Th cell populations in the blood and lymphoid tissues, and lower Th cell activation in PPs. These findings were compatible with previous reports that sepsis results in lymphocyte depletion and impairment of T-cell function. APS administration reversed sepsis-induced decrements in Th cell populations in the blood and spleen at the higher dose of APS. Also, the percentage of activated Th cells in the spleen and PPs increased when APS was administered. These results indicated that APS prevent depletion of Th cells and enhance activation of Th cells in sepsis.

Th cells and Treg play important roles in the proper development of cellular and humoral immune responses during sepsis. Sepsis-induced abnormalities of CD4 T-cell subpopulations were reported, including polarization to the Th2 subset and increments of Treg populations [2]. Th1 cells produce IFN- $\gamma$ which activates macrophages to kill intracellular microorganisms, whereas Th2 cells secrete IL-4 which enhances the B-cell response and alternative activation of macrophages to promote tissue repair [8]. IFN- $\gamma$ and IL4 also, respectively, act in an autocrine loop on differentiating Th1 and Th2 cells. The shift from a proinflammatory Th1 phenotype to an anti-inflammatory Th2 phenotype is regulated by mitogen-activated protein kinases (MAPKs). A study by Song et al. [26] reported that induction of p38 MAPK activation in splenic lymphocytes contributes to the immunosuppression seen in septic mice. In the present study, the percentage of IL-4-expressing Th cells in the blood was higher and the IFN- $\gamma / \mathrm{IL}-4$ ratio was lower in the saline group, indicating that Th cells had shifted toward a Th2type response. Splenic cytokine mRNA expressions were consistent with these results that Th1-related cytokines were lower and Th2 cytokines were higher than those of the sham group. APS administration downregulated circulating Th2 cells, inhibited the Th phenotype toward the Th2 response in the blood, and suppressed splenic IL-4 mRNA expression. We also observed that the sepsis-induced decrement in intestinal IgA was partially reversed in the A200 and A400 groups, suggesting that although APS administration inhibited Th2 polarization, the function of IgA secretion was not impaired. Our results suggested that a more balanced Th1/Th2 response occurred during sepsis when APS was provided. A previous study indicated that APS induced the differentiation of splenic dendritic cells and further modulated the immune function of Th cells with a shift from Th2 to Th1 [17]. 

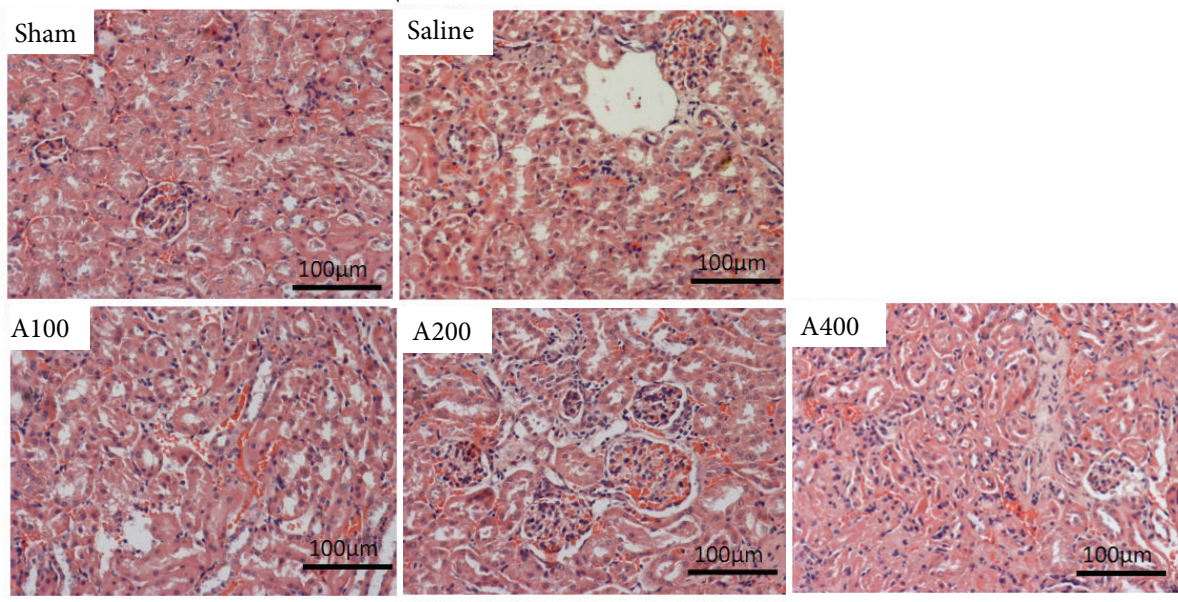

(a)

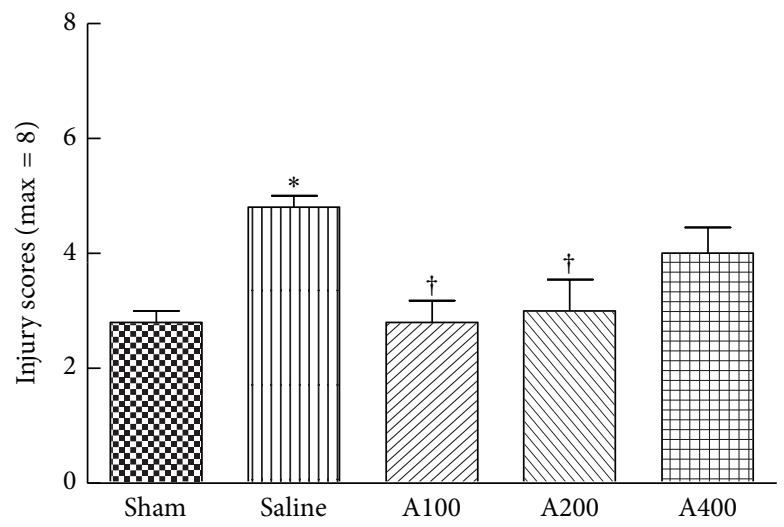

(b)

FIGURE 5: Histopathology of the kidney. Representative histological images are shown at 200x magnification. (a) Kidney tissues stained with hematoxylin and eosin. (b) Histological scores of kidney injury are presented as the mean \pm SEM, which were determined as described in Section $2 .{ }^{*}$ Significantly different from the sham group $(p<0.05)$. ${ }^{\dagger}$ Significantly different from the saline group $(p<0.05)$.

Th17 cells, the major source of IL-17A, facilitate neutrophil recruitment and promote clearance of extracellular pathogens by neutrophils [27]. However, the importance of Th17 cell effector functions in sepsis is still under investigation. Flierl et al. [28] indicated that plasma IL-17A increased after CLP surgery, which was mainly derived from $\gamma \delta \mathrm{T}$ cells, and IL-17A promoted high levels of proinflammatory cytokines, resulting in negative outcomes in experimental sepsis. Treg are involved in suppressing excessive T-cell responses, and Foxp3 is a key transcription factor in Treg development and function [9]. Treg are more resistant to sepsis-induced apoptosis than are other effector CD4 T cells [29], which explains the increased percentage of circulating Treg in patients with sepsis [10]. Our results indicated that the percentage of IL-17A-expressing Th cells in the blood was not affected by sepsis, although IL-17A mRNA expression was downregulated in the spleen. Since Th17 cells are not the only source of IL-17A [27], further studies are required to clarify the roles of IL-17A-producing cells in sepsis. A higher blood Treg percentage was also observed in the saline group, which is compatible with a previous report [10].
In this study, declines in IL-17A and IL-6 mRNA expressions in spleen were prevented by APS administration. Treatment with the high dose of APS even upregulated transcription levels of splenic IL-17A and IL- 6 mRNA and the population of blood Th17 cells. IL-6 has counter-regulatory effects in modulating Treg and Th17 differentiation from naive T cells. IL- 6 promotes the differentiation of Th17 cells together with TGF- $\beta$ [30]. However, Treg differentiation is inhibited by IL-6 [31], which could explain the findings of lower Foxp3 mRNA expression and circulating Treg frequencies in the APS-treated groups. The Treg-suppressing effects of APS were demonstrated in different animal models [16, 32]. In a murine model of burns with bacterial infection, APS was found to suppress the expression of Foxp 3 by $\mathrm{CD} 4{ }^{+} \mathrm{CD} 25^{+}$ Treg and to activate Thl cell-mediated immunity [16]. Those authors concluded that the influence of APS on Treg was possibly partially mediated by binding the Toll-like receptor 4 on the surface of Treg. Our results suggested that modulation of cytokine profiles by APS also plays important roles in Th and Treg polarization. 
Multiple organ failure is a critical complication of sepsis, and sepsis-induced renal dysfunction is associated with the high mortality rate [33]. In order to understand the impact of APS on organ injury, morphological changes and injury scores of kidney tissues were analyzed. The histological findings showed that sepsis resulted in damage to tubular cells, but the damage to the kidneys was less severe in the septic groups with dosages of 100 and $200 \mathrm{mg}$ APS $/ \mathrm{kg}$ of BW. Since APS administration leads to a more balanced Th/Treg response, this change may subsequently improve sepsisinduced tissue injury. However, kidney injury was not attenuated with APS administration at a dosage of $400 \mathrm{mg}$ APS $/ \mathrm{kg}$ of BW, which should be due to excessive polarization toward the proinflammatory Thl and Th17 lineages. Previous studies have indicated that both Thl and Th17 cells are capable of inducing renal inflammation and injury $[34,35]$.

In summary, this study showed that APS treatment at dosages of 100 and $200 \mathrm{mg} / \mathrm{kg}$ of BW increased Th cell percentages in the circulation and lymphoid tissues, decreased the percentage of Treg, and inhibited the polarization of blood $\mathrm{CD} 4^{+} \mathrm{T}$ cells toward a Th2 response that may consequently attenuate kidney injury in antibiotic-treated septic mice. However, high-dose APS administration may excessively reverse the dysregulated $\mathrm{Th} / \mathrm{Treg}$ response and have adverse effects in sepsis-induced organ injury. We conclude that APS is an immunopotentiator and proper dosage of APS may be a potential adjuvant treatment for sepsis-induced immunosuppression.

\section{Conflict of Interests}

The authors declare that they have no conflict of interests.

\section{Acknowledgments}

The authors would like to thank Man-Hui Pai, Associate Professor of Department of Anatomy and Cell Biology, Taipei Medical University, for her technical assistance in the preparation of histological examination. This study was supported by a research grant (NSC 102-2314-B-002-090MY3) from the National Science Council, Taipei, Taiwan.

\section{References}

[1] R. P. Dellinger, M. M. Levy, A. Rhodes et al., "Surviving sepsis campaign: international guidelines for management of severe sepsis and septic shock: 2012," Critical Care Medicine, vol. 41, no. 2, pp. 580-637, 2013.

[2] R. S. Hotchkiss, G. Monneret, and D. Payen, "Sepsisinduced immunosuppression: from cellular dysfunctions to immunotherapy," Nature Reviews Immunology, vol. 13, no. 12, pp. 862-874, 2013.

[3] J.-L. Vincent, D. R. Nelson, and M. D. Williams, "Is worsening multiple organ failure the cause of death in patients with severe sepsis?" Critical Care Medicine, vol. 39, no. 5, pp. 1050-1055, 2011.

[4] G. P. Otto, M. Sossdorf, R. A. Claus et al., "The late phase of sepsis is characterized by an increased microbiological burden and death rate," Critical Care, vol. 15, no. 4, article R183, 2011.
[5] R. S. Hotchkiss, K. W. Tinsley, P. E. Swanson et al., "Sepsisinduced apoptosis causes progressive profound depletion of $\mathrm{B}$ and $\mathrm{CD} 4^{+} \mathrm{T}$ lymphocytes in humans," Journal of Immunology, vol. 166, no. 11, pp. 6952-6963, 2001.

[6] Y. Le Tulzo, C. Pangault, A. Gacouin et al., "Early circulating lymphocyte apoptosis in human septic shock is associated with poor outcome," Shock, vol. 18, no. 6, pp. 487-494, 2002.

[7] J. S. Boomer, K. To, K. C. Chang et al., "Immunosuppression in patients who die of sepsis and multiple organ failure," The Journal of the American Medical Association, vol. 306, no. 23, pp. 2594-2605, 2011.

[8] J. Cabrera-Perez, S. A. Condotta, V. P. Badovinac, and T. S. Griffith, "Impact of sepsis on CD4 T cell immunity," Journal of Leukocyte Biology, vol. 96, no. 5, pp. 767-777, 2014.

[9] S. Sakaguchi, "Naturally arising Foxp3-expressing $\mathrm{CD} 25^{+} \mathrm{CD} 4^{+}$ regulatory $\mathrm{T}$ cells in immunological tolerance to self and nonself," Nature Immunology, vol. 6, no. 4, pp. 345-352, 2005.

[10] G. Monneret, A.-L. Debard, F. Venet et al., "Marked elevation of human circulating CD4+CD25+ regulatory T cells in sepsisinduced immunoparalysis," Critical Care Medicine, vol. 31, no. 7, pp. 2068-2071, 2003.

[11] F. Venet, C.-S. Chung, H. Kherouf et al., "Increased circulating regulatory $\mathrm{T}$ cells $\left(\mathrm{CD} 4^{+} \mathrm{CD} 25^{+} \mathrm{CD} 127^{-}\right)$contribute to lymphocyte anergy in septic shock patients," Intensive Care Medicine, vol. 35, no. 4, pp. 678-686, 2009.

[12] D. McKenna, K. Hughes, and K. Jones, “Astragalus," Alternative Therapies in Health and Medicine, vol. 8, no. 6, pp. 34-40, 2002.

[13] X. Q. Ma, Q. Shi, J. A. Duan, T. T. X. Dong, and K. W. K. Tsim, "Chemical analysis of Radix Astragali (Huangqi) in China: a comparison with its adulterants and seasonal variations," Journal of Agricultural and Food Chemistry, vol. 50, no. 17, pp. 4861-4866, 2002.

[14] K. S. Zhao, C. Mancini, and G. Doria, "Enhancement of the immune response in mice by Astragalus membranaceus extracts," Immunopharmacology, vol. 20, no. 3, pp. 225-233, 1990.

[15] B.-M. Shao, W. Xu, H. Dai, P. Tu, Z. Li, and X.-M. Gao, "A study on the immune receptors for polysaccharides from the roots of Astragalus membranaceus, a Chinese medicinal herb," Biochemical and Biophysical Research Communications, vol. 320, no. 4, pp. 1103-1111, 2004.

[16] Q. Y. Liu, Y. M. Yao, Y. Yu et al., “Astragalus polysaccharides attenuate postburn sepsis via inhibiting negative immunoregulation of $\mathrm{CD} 4^{+} \mathrm{CD} 25^{\text {high }}$ T cells," PLoS ONE, vol. 6, no. 6, Article ID e19811, 2011.

[17] Q.-Y. Liu, Y.-M. Yao, S.-W. Zhang, and Z.-Y. Sheng, "Astragalus polysaccharides regulate $T$ cell-mediated immunity via CD11 $\mathrm{c}^{\text {high }} \mathrm{CD} 45 \mathrm{RB}^{\text {low }}$ DCs in vitro," The Journal of Ethnopharmacology, vol. 136, no. 3, pp. 457-464, 2011.

[18] M. Kuruş, M. Ugras, and M. Esrefoglu, "Effect of resveratrol on tubular damage and interstitial fibrosis in kidneys of rats exposed to cigarette smoke," Toxicology and Industrial Health, vol. 25, no. 8, pp. 539-544, 2009.

[19] W. C. S. Cho and K. N. Leung, "In vitro and in vivo immunomodulating and immunorestorative effects of Astragalus membranaceus," The Journal of Ethnopharmacology, vol. 113, no. 1, pp. 132-141, 2007.

[20] H. Wei, R. Sun, W. Xiao et al., "Traditional Chinese medicine Astragalus reverses predominance of Th2 cytokines and their up-stream transcript factors in lung cancer patients," Oncology Reports, vol. 10, no. 5, pp. 1507-1512, 2003. 
[21] H.-W. Chen, I.-H. Lin, Y.-J. Chen et al., "A novel infusible botanically-derived drug, PG2, for cancer-related fatigue: a phase II double-blind, randomized placebo-controlled study," Clinical and Investigative Medicine, vol. 35, no. 1, pp. E1-E11, 2012.

[22] S. Reagan-Shaw, M. Nihal, and N. Ahmad, "Dose translation from animal to human studies revisited," The FASEB Journal, vol. 22, no. 3, pp. 659-661, 2008.

[23] A. Ayala and I. H. Chaudry, "Immune dysfunction in murine polymicrobial sepsis: mediators, macrophages, lymphocytes and apoptosis," Shock, vol. 6, supplement 1, no. 4, pp. S27-S38, 1996.

[24] D. Rittirsch, M. S. Huber-Lang, M. A. Flierl, and P. A. Ward, "Immunodesign of experimental sepsis by cecal ligation and puncture," Nature Protocols, vol. 4, no. 1, pp. 31-36, 2009.

[25] R. S. Hotchkiss, R. E. Schmieg Jr., P. E. Swanson et al., "Rapid onset of intestinal epithelial and lymphocyte apoptotic cell death in patients with trauma and shock," Critical Care Medicine, vol. 28, no. 9, pp. 3207-3217, 2000.

[26] G. Y. Song, C.-S. Chung, I. H. Chaudry, and A. Ayala, "Immune suppression in polymicrobial sepsis: differential regulation of Th1 and Th2 responses by p38 MAPK," Journal of Surgical Research, vol. 91, no. 2, pp. 141-146, 2000.

[27] D. J. Cua and C. M. Tato, "Innate IL-17-producing cells: the sentinels of the immune system," Nature Reviews Immunology, vol. 10, no. 7, pp. 479-489, 2010.

[28] M. A. Flierl, D. Rittirsch, H. Gao et al., "Adverse functions of IL-17A in experimental sepsis," The FASEB Journal, vol. 22, no. 7, pp. 2198-2205, 2008.

[29] F. Venet, A. Pachot, A.-L. Debard et al., "Increased percentage of $\mathrm{CD} 4^{+} \mathrm{CD} 25^{+}$regulatory $\mathrm{T}$ cells during septic shock is due to the decrease of $\mathrm{CD} 4^{+} \mathrm{CD} 25^{-}$lymphocytes," Critical Care Medicine, vol. 32, no. 11, pp. 2329-2331, 2004.

[30] E. Bettelli, Y. Carrier, W. Gao et al., "Reciprocal developmental pathways for the generation of pathogenic effector TH17 and regulatory T cells," Nature, vol. 441, no. 7090, pp. 235-238, 2006.

[31] T. Korn, M. Mitsdoerffer, A. L. Croxford et al., "IL-6 controls Th17 immunity in vivo by inhibiting the conversion of conventional T cells into Foxp3+ regulatory T cells," Proceedings of the National Academy of Sciences of the United States of America, vol. 105, no. 47, pp. 18460-18465, 2008.

[32] X. Du, X. Chen, B. Zhao et al., "Astragalus polysaccharides enhance the humoral and cellular immune responses of hepatitis $B$ surface antigen vaccination through inhibiting the expression of transforming growth factor $\beta$ and the frequency of regulatory T cells," FEMS Immunology and Medical Microbiology, vol. 63, no. 2, pp. 228-235, 2011.

[33] J. A. Russell, J. Singer, G. R. Bernard et al., "Changing pattern of organ dysfunction in early human sepsis is related to mortality," Critical Care Medicine, vol. 28, no. 10, pp. 3405-3411, 2000.

[34] H.-J. Paust, J.-E. Turner, O. M. Steinmetz et al., “The IL-23/Th17 axis contributes to renal injury in experimental glomerulonephritis," Journal of the American Society of Nephrology, vol. 20, no. 5, pp. 969-979, 2009.

[35] S. A. Summers, O. M. Steinmetz, M. Li et al., "Th1 and Th17 cells induce proliferative glomerulonephritis," Journal of the American Society of Nephrology, vol. 20, no. 12, pp. 2518-2524, 2009. 


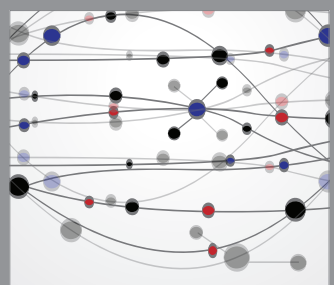

The Scientific World Journal
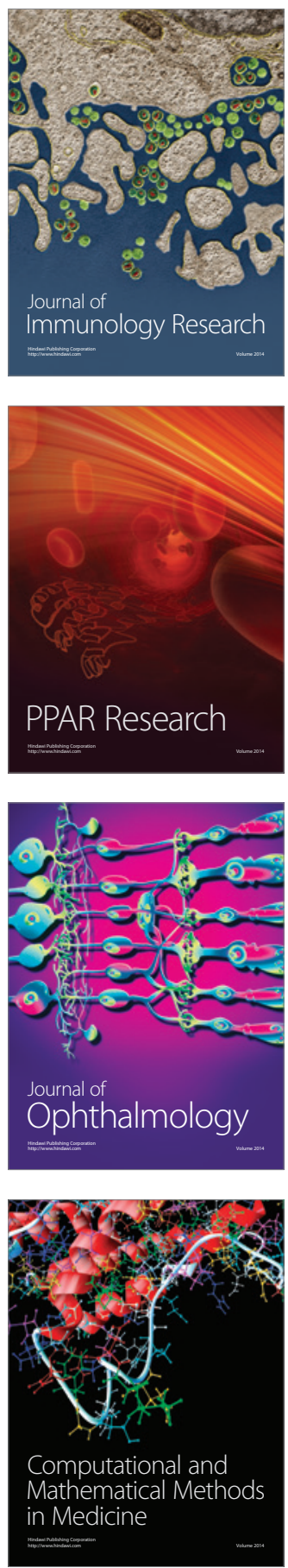

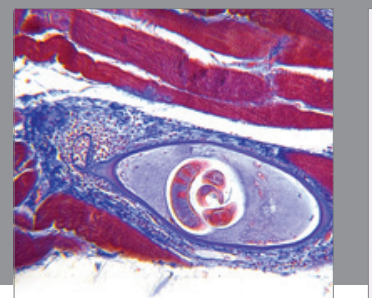

Gastroenterology

Research and Practice
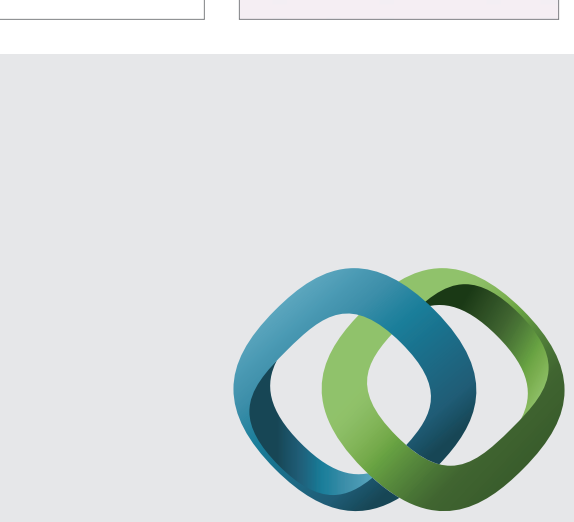

\section{Hindawi}

Submit your manuscripts at

http://www.hindawi.com
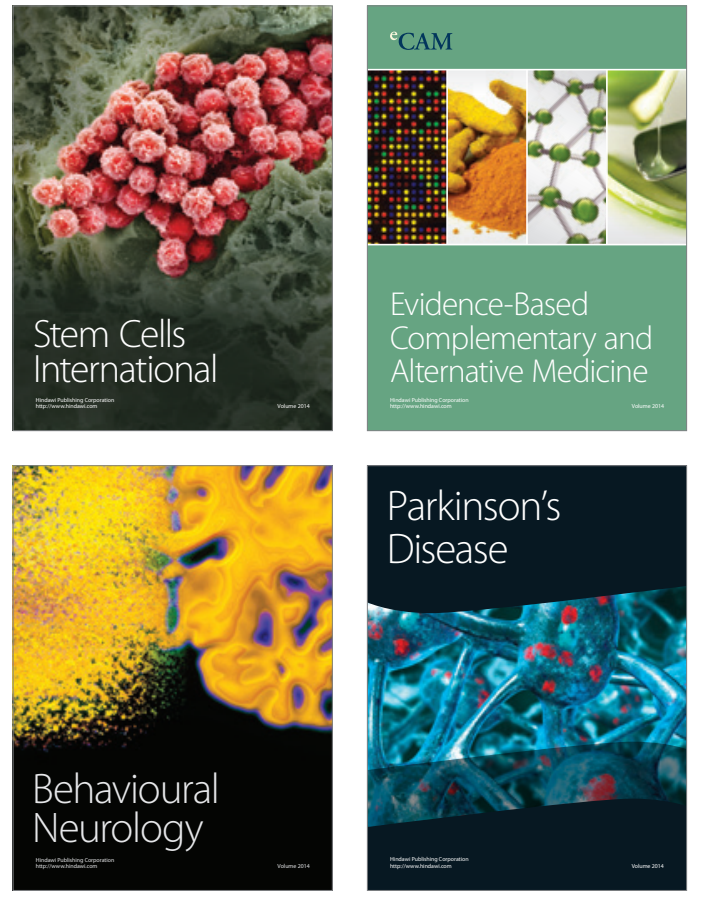
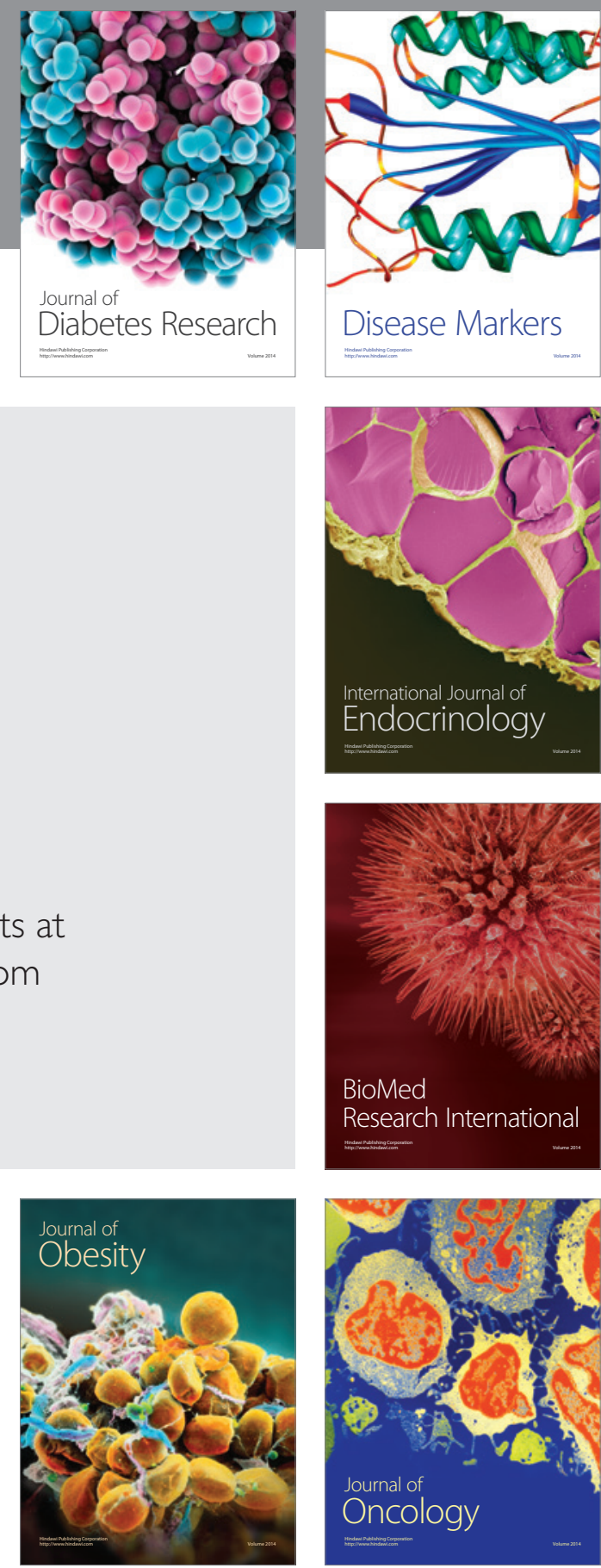

Disease Markers
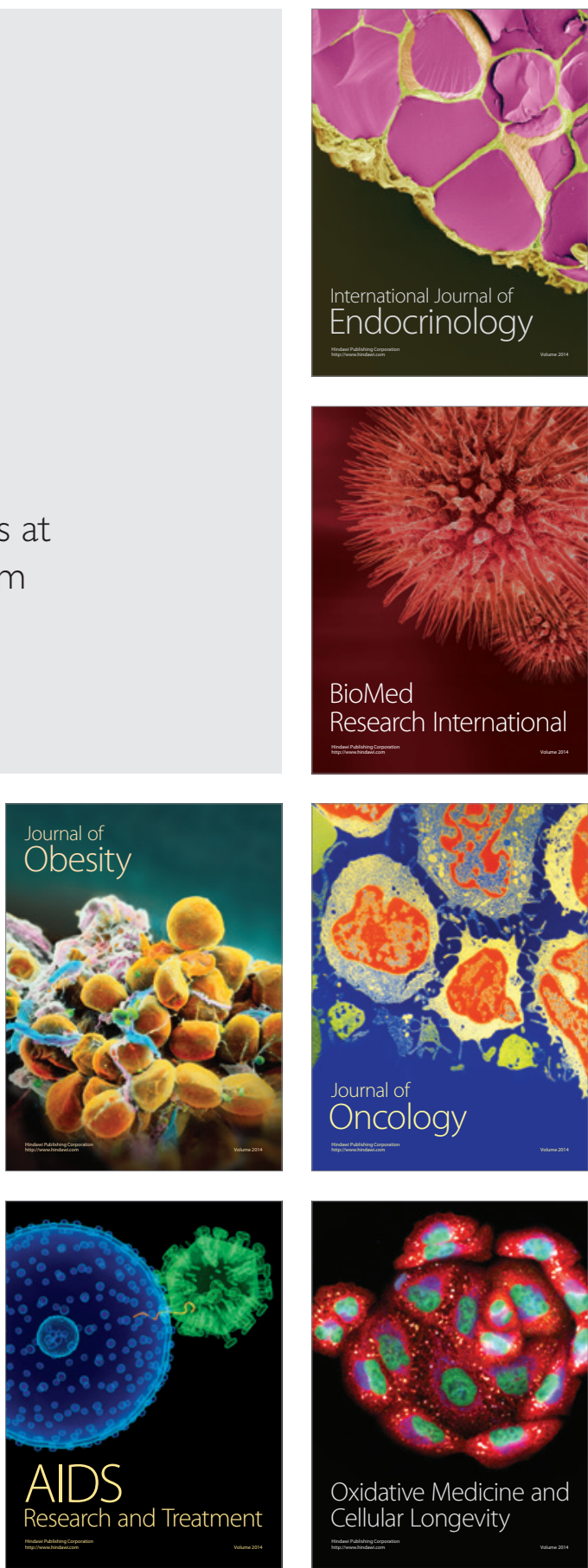